\title{
ECG Monitoring Using Android Mobile Phone and Bluetooth
}

\author{
Samuel E. de Lucena, Daniel J. B. S. Sampaio \\ Electrical Engineering Department \\ Unesp - Sao Paulo State University \\ Guaratingueta, Brazil \\ sdelucena@gmail.com
}

\author{
Benjamin Mall, Michael Meyer, Martin A. Burkart, \\ Frieder V. Keller \\ Fakultät für Elektro- und Informationstechnik \\ University of Applied Science \\ Karlsruhe, Germany \\ frieder.keller@hs-karlsruhe.de
}

\begin{abstract}
This paperwork describes the development and test of circuitry and software to enable the use of Android mobile phones equipped with Bluetooth to receive the incoming electrocardiogram (ECG) signal from a user and show it in realtime on the cell phone screen. The system comprises three distinct subsystems. The first one is dedicated to condition the analog ECG signal, preparing it for conversion to the digital world. The second one consists of a microcontroller and a Bluetooth module. This unit samples the ECG, serializes the samples and transmits them via the Bluetooth module to the Android cell phone. The third subsystem is the cell phone itself. An application program written to the cell phone receives the ECG samples and suitably charts the ECG signal on the screen for analysis. The good quality of the ECG signal allows for identification of arrhythmias.
\end{abstract}

Keywords- ECG; Android; Bluetooth; cardiac patients; wireless medical applications; home healtcare; embedded systems

\section{INTRODUCTION}

Ischemic heart disease has remained one of the top killers worldwide during the last decade. According to the World Health Organization (WHO), in 2002 only, 7.2 million people died from coronary heart disease [1]. While life style and food choices, among other factors, have an influence on this death toll, it is undisputable that broad access to quality healthcare services would increase population's probability to avoid, survive or yet live longer with heart disease (and other illnesses, of course).

ECG monitoring is effective in both forecasting heart disease and keeping cardiac patients under tight surveillance of their heart conditions [2]. Widespread use of ECG monitoring is, however, a pretty distant goal, mainly in nations of the developing world and poor nations. The main reasons for that are the high cost of ECG machines, bulky ECG equipment, the need for a specialist to operate the ECG device, and the competing demands from other areas (e.g. educational and infrastructure development) for limited national budgets. Indeed, significant part of population within the nations of industrialized world cannot afford private healthcare. Therefore, reducing costs of medical devices can lead to improvement of the aforementioned scenario.

This work was supported by Capes (Coordination for the Improvement of Higher Level Education; Brazil) and DAAD (Deutscher Akademischer Austauschdienst; Germany).
One way to cut down cost of ECG monitoring devices is to implement these around ubiquitous mobile phones (and tablets) running under Android operating system and equipped with wireless Bluetooth technology. The following benefits may result from the massive adoption of this technology, besides lowering ECG monitoring cost. Patients may have their ECG recorded at home, avoiding transporting to distant hospitals and moving though heavy traffic urban areas. This might be quite convenient for elderly patients, chronic cardiac patients, and patients living in the countryside where doctors are not available. Perhaps this explains why home healthcare is the fastest-growing segment of the medical device industry, according to Food and Drug Administration (FDA) [3].

In addition to replacing expensive and bulky traditional ECG machines, mobile phone-based ECG monitoring devices offer the paramount feature of instant warning about the heart condition of the patient. This characteristic is quite appealing, for life threatening arrhythmias and ECG alterations appear before a sudden heart attack occurs. Moreover, the chance to survive such an event is higher when patients are treated promptly [4].

Whereas initial research efforts favoring mobile ECG monitoring utilized personal data assistants (PDA) handhelds [5], and cell phones to transmit ECG data to personal computers (PC) [6], most researchers clearly shifted to mobile cell phones operating under Android operating system and equipped with Bluetooth wireless technology [3], [4], [7].

This paper presents the design and evaluation of an ECG monitoring system deploying an Android mobile phone and Bluetooth technology. Paper organization is as follows: section II describes the system architecture and design methodology. Section III shows the results and discussion, whereas section IV contains the conclusion.

\section{SYSTEM ARCHITECTURE AND METHODOLOGY}

\section{A. System Overview}

The designed ECG monitoring system comprises three distinct subsystems, as depicted in Fig. 1. The first one is dedicated to process the analog ECG signal, preparing it for conversion to the digital world. This is necessary, for today's 
mobile phones do not include means to directly interface to analog signals from the external world. The second subsystem consists of a microcontroller and a Bluetooth module. This unit samples the ECG, serializes the samples and transmits them via the Bluetooth module to the Android cell phone. The third subsystem is the cell phone itself. An application program written to the cell phone receives the ECG samples and suitably plots the ECG signal on the screen for analysis. Fig. 2 shows a picture of a volunteer connected to the ECG monitoring system. One can easily identify the system`s parts in the picture of Fig. 3.

Fig. 4 presents the schematics of the ECG conditioning circuit. Power supply decoupling capacitors are not shown, for the sake of simplicity. An instrumentation amplifier (IA) (Texas Inst., INA101) receives the ECG detected by $\mathrm{Ag}-\mathrm{AgCl}$ surface electrodes located on the patient's right arm (RA), left arm (LA) and right leg (RL), and uses a resistor to set its gain to an appropriate value. This was experimentally adjusted. RL is the reference electrode. This electrode combination is named lead I, but any other lead of the classic 12-lead ECG might have been used. Using shielded cables to connect with the LA and RA electrodes significantly improved the signal to noise ratio (SNR). Two 9-V batteries supply symmetrical voltages to IA and all the system's operational amplifiers (Op Amps).

The amplified ECG signal, as it appears at the output of the IA (pin 1), first goes through a simple RC high-pass filter (HPF). This passive filter's cutoff frequency is about $0.23 \mathrm{~Hz}$ and its role is to block the unwanted high-amplitude dccomponent of the signal. To filter out the main's $60-\mathrm{Hz}$ interference noise on the ECG signal, this is buffered and then processed by a $60-\mathrm{Hz}$ notch filter. The voltage follower is built around a precision Op Amp (Texas Inst., OP27), while the notch filter deployed one Op Amp (A) from classic LM324 IC (Texas Inst.) containing four Op Amps. To limit the signal's bandwidth, and avoid errors due to aliasing during the sampling process, now the signal is processed by a secondorder, Butterworth active low-pass filter (LPF) that sets the cutoff frequency to $482 \mathrm{~Hz}$. This active LPF further provides a voltage gain of 1.586 and is assembled around one more (B) of LM234's four Op Amps. As long as the microcontroller's analog-to-digital converter (ADC) input signal spans from zero to $3.3 \mathrm{~V}$, a final analog signal processing task consisting in shifting the signal so that it fits suitably to the ADC's input voltage range is needed. This is carried out by the third (C) Op Amp from LM324, which is arranged as a summing circuit. The appropriate offset voltage level is adjusted using the 500 $\mathrm{k} \Omega$ trimpot.

Several different processing circuits could have been utilized to condition the ECG signal. For instance, (digitally) programmable-gain IA could be quite interesting an option. Also, choosing to eliminate the $60-\mathrm{Hz}$ interference noise deploying a digital filter can be quite appealing, given that very high-order digital filters are easily constructed and implemented with modern microcontrollers. However, the circuit shown in this work, other than effective, enables the development team to readily modify it without the need to rewrite, compile and download control programs to the MCU.

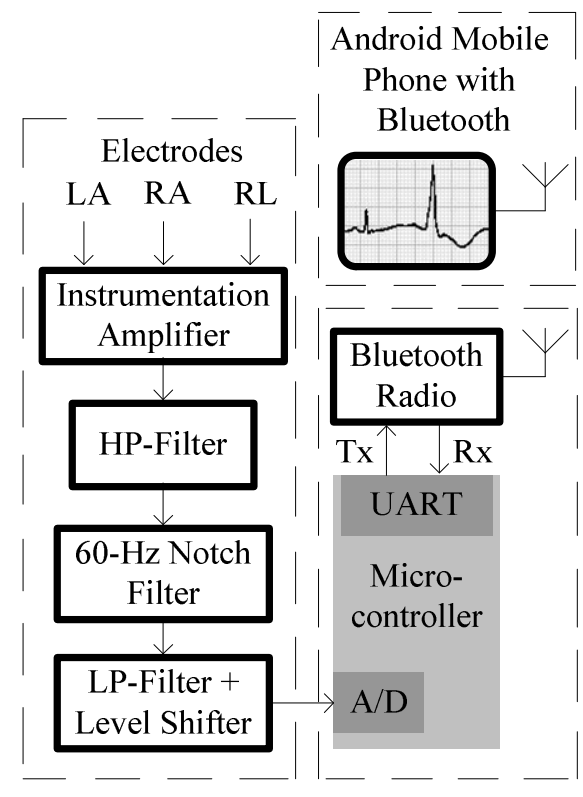

Fig. 1. Android mobile phone-based ECG monitoring system.

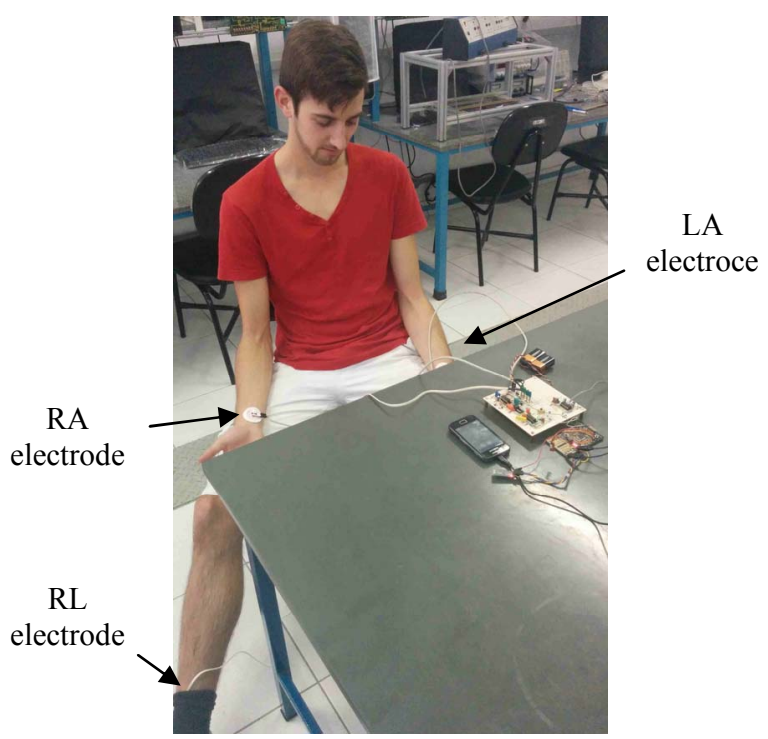

Fig. 2. Mobile phone-based ECG monitoring prototype connected to a user.

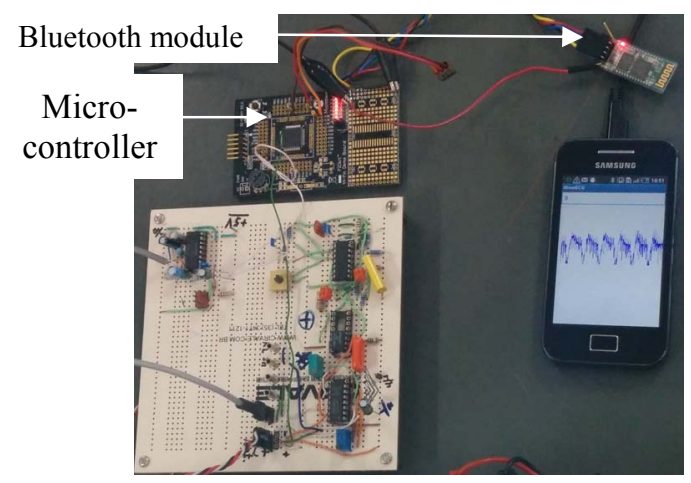

Fig. 3. Close-up of the mobile phone-based ECG monitoring system. 


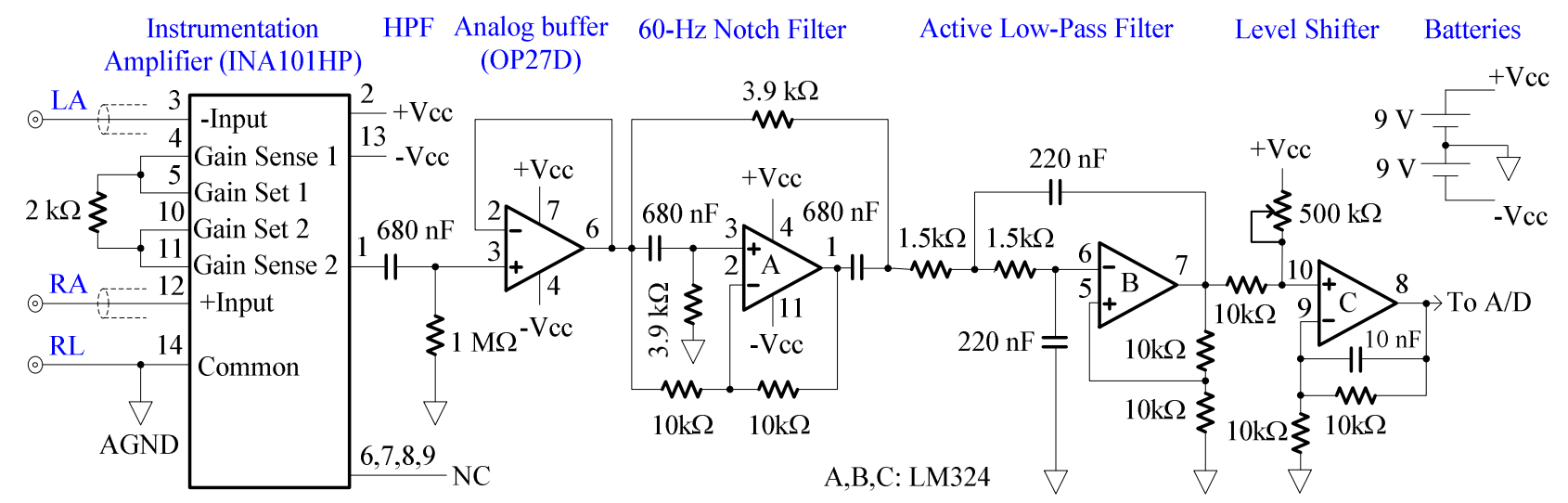

Fig. 4. ECG conditioning circuitry.

\section{B. ECG Sampling and Transmission via Bluetooth}

An 8-bit microcontroller (Microchip, PIC18F45k20) endlessly samples the ECG signal at $150 \mathrm{~Hz}$, using an embedded 10-bit ADC. The control program, written in $\mathrm{C}$ language, sends the incoming raw samples to an embedded USART serial port. The program reduces every 10-bit sample into a correspondent 8-bit sample, before transmission, simply by discarding the two less significant bits. The USART serializes the samples at 9600 bits per seconds, using the following settings: 8-bit data length, no parity, and one stop bit. Upon receiving the bits streaming from the USART, the Bluetooth module (Linvor JY-MCU) sends them into the air, which can be received by a nearby Bluetooth-equipped mobile phone. Pairing, however, of the Bluetooth module with the mobile phone's Bluetooth must have been accomplished before any data transfer can take place. The control program running on the microcontroller just implements the commands supplied by the datasheet for the Bluetooth module (EGBT046S AT Command Set) to establish communication with the mobile phone. The used module is a class 2 radio $(2.5-\mathrm{mW}$ power and 10-m range).

\section{Android Mobile Phone}

A commercially available Android mobile phone with Bluetooth (Samsung's Galaxy Ace, GT-S5830B, Fig. 3) served as the target mobile phone during the implementation of this research project. At first, App Inventor (www. appinventor.mit.edu) served as the programming language and IDE for this research project. It worked fully and correctly, but as it had to be run on-line and our laboratory began to face Internet connection instability, our group decided to switch to a desktop-based, free and yet quite powerful IDE and compiler. We adopted Eclipse IDE (www.eclipse.org) and rewrote the entire Android application for the mobile phone utilizing the Java compiler of GCC (www.gcc.gnu.org).

The ever increasing stunning processing power of the 32bits microcontrollers populating mobile phones (virtually almost $100 \%$ of these microcontrollers are manufactured under license of ARM Group) has opened up the possibility of inclusion of very complex signal processing algorithms into the applications programs. Furthermore, for Android-powered mobile phones, as is the case in this study, this application inclusion into the mobile is quite straightforward and easy. In this research project, however, the application program is dedicated to receiving the ECG samples through the Bluetooth radio and processing them to properly plot the ECG trace on the mobile's screen.

\section{RESULTS AND DISCUSSION}

Two distinct series of tests have been carried out to assess the performance of the designed ECG monitoring system. The next two subsections describe them in detail.

\section{A. Prototype Evaluation Using an Arrhythmia Simulator}

One effective method to evaluate how well a waveform recording instrument performs is to inject known signals into it and then examining its output, in this case, the quality of the recorded waveforms. The test signals must be of high quality and span across all the frequency and amplitude values the instrument under test is supposed to face in real world. An industry standard ECG and arrhythmia simulator has been deployed to assess the prototype's adequacy as an ECG recording and monitoring system. The utilized simulator is capable of synthesizing waveforms of physiological quality and is used by the industry either as a development tool or as a service tool (for instance, to test automatic arrhythmia detecting systems). The simulator has been connected to the prototype's electrodes (RA, LA and RL) and recording of more than two dozen different normal and arrhythmic ECG waveforms have been carried out by the prototype. TABLE I contains a selection of the waveforms recorded by the prototype. These waveforms have been captured directly from the mobile phone's screen into a notebook, as image files, by means of a function on the integrated development environment (Eclipse IDE).

The ECGs in TABLE I are identified using standard arrhythmia nomenclature. As could be noted, the prototype was capable of recording the several ECG waveforms with a high degree of fidelity. This could be observed by comparing the simulator's signals with the corresponding prototype's output signals. As an expert reader can easily observe, the prototype enables good quality recording of all key waveform details that are of paramount importance in ECG analysis (i.e., characteristic $\mathrm{P}$ and $\mathrm{T}$ waves and both normal and abnormal 
QRS complexes). One observed degradation in the sampled waveforms showed up as input signal saturation. This can be viewed in the recordings of premature ventricular contraction (PVC 2) and R-on-T phenomenon arrhythmias, where the typical V-like waves appear somewhat rounded on the vertex. Solutions to this problem include decreasing amplifier gain, and adjusting the offset voltage added up to the signal just before the ADC.

\section{B. Prototype Evaluation Using Individuals}

This subsection contains some results of the prototype qualitative evaluation via the ECG recording of several individuals. One person at a time had the electrodes connected to his right leg, right arm and left arm, and his ECG recorded. During the recordings, individuals remained sitting on a chair and were asked to keep as relaxed as possible (as illustrated in Fig. 2). Their ages and weights span from 22 to 52 years, and from 65 to $93 \mathrm{~kg}$, respectively. They were all males, with no reported health problems.

Fig. 5 is a snapshot (into the Eclipse IDE) showing how the first application screen looks like on the mobile phone's screen. The user counts on the buttons named Bluetooth and $E C G$ to connect the mobile phone with the ECG module's Bluetooth and to acquire the ECG signal, respectively. So far, Sp02, Blood Pressure and Temperature functions have not been implemented.

Fig. 6(a) is a direct photograph of the mobile`s screen while volunteer identified as 1 was kept connected to the ECG electrodes and a recording section was commanded. This procedure has been repeated with several volunteers, but rather than photographing the mobile's screen, screen shots have been captured by Eclipse IDE, for documentation purposes. Figs. 6(b) through (d) present these screen shots for three different individuals, identified here as 2, 3 and 4, respectively. Overall, the ECG traces are clear enough to allow their analysis whenever needed. In particular, the signal quality is suitable for monitoring purpose.

Different types of noise (e.g., $60-\mathrm{Hz}$ interference from power grid) can easily contaminate and destroy the milivoltlevel ECG signals during recordings. Fig. 6(e) shows the ECG of one individual, recorded using the assembled prototype, with strong interference noise. As a rule, this type of distortion may be avoided by means of adequate connection of cables, maintaining these cables at a distance from power cables, and instructing the person using the instrument to be sitting in a relaxed posture. Moreover, though not yet implemented in this project, digital filters running inside the mobile phone (as part of the ECG application) can be quite effective in wiping out several noise types.

\section{CONCLUSION}

Thanks to the stunning processing power of the microcontrollers embedded in mobile phones, the relative low cost of these genre of equipment, the high resolution of their displays, the widespread use of Android operating system, and the existence of powerful and yet free, open-source IDE and compilers, Android mobile phones will almost inexorably be part of the home healthcare scenario in the coming years.

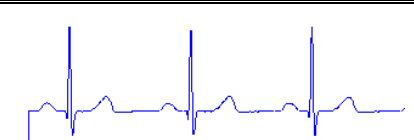

Normal sinus rhythm (60 BPM)

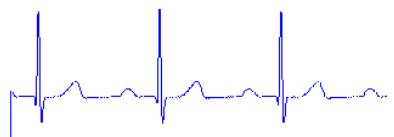

First-degree atrioventricular (AV) block (60 BPM)
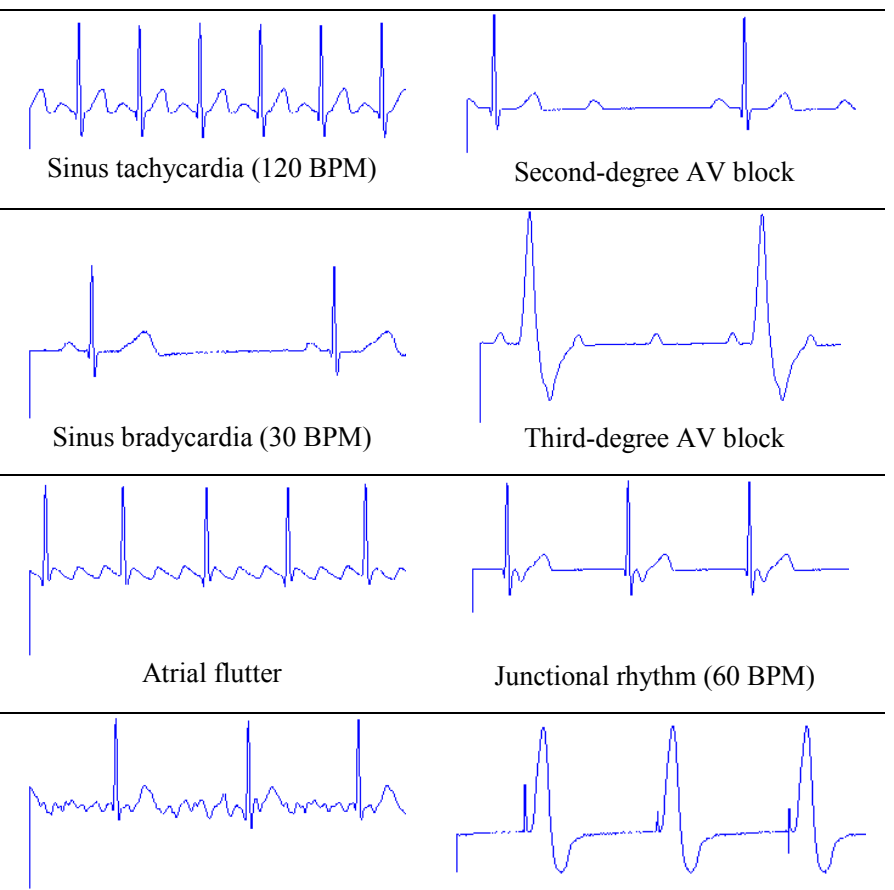

Atrial fibrillation

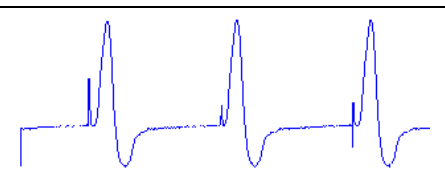

Asynchronous ventricular pacemaker (60 BPM)

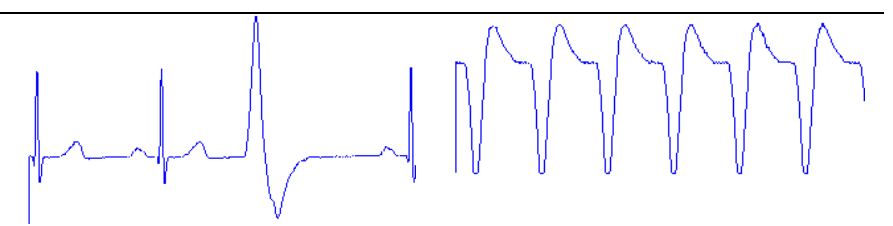

Premature ventricular contraction (PVC 1)

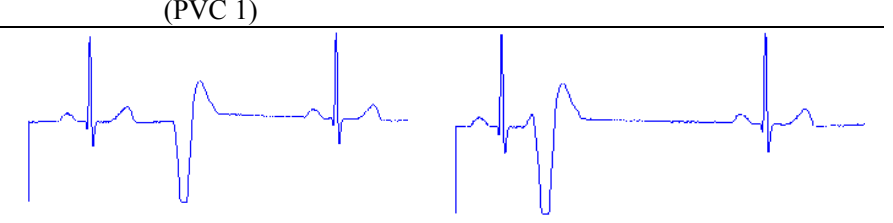

Premature ventricular contraction (PVC 2)

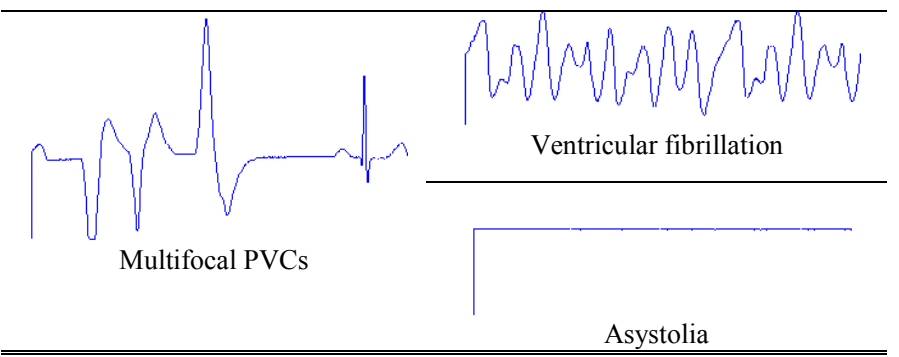


As discussed earlier, this can bring a series of advantages to both the patients and healthcare systems. Lowering costs and easing patient lives are the two chief advantages. Much research is conducted, for instance, to assess the feasibility of these mobile phones as ECG monitoring equipments. In this case, patients will benefit from the fact that they can have instant recording of their ECGs and this can be of paramount importance, as long as the chance to survive a serious cardiac event decreases as time to take action increases. Another very appealing feature of this technology shift is that it can save much time avoiding patients' transportation to hospitals or doctor offices to have their exams done. For elderly and chronic cardiac patients this is quite an attractive feature. Population living in rural areas and small villages will have access to specialists working in big cities.

This paper presented a prototype for ECG monitoring based on mobile phone technology which performed quite well with both simulated normal and abnormal ECGs stemming from an ECG and arrhythmia simulator. Overall, the prototype could present good quality ECG traces on the mobile's screen when connected to several individuals. The good results achieved with the presented prototype ignited the enthusiasm of our group to further improve the project. We believe that the following features are mandatory:

- Implementation of a heart rate meter function. This simple function can promptly inform the patient and caretakers about the patient's heartbeat frequency.

- Implementation of a 12-lead acquisition and display function. This is fundamental to enable diagnostic on the heart muscle by a specialist. The patient or a caretaker can select the equipment operating mode: one-lead monitoring or 12-lead recording.

- Developing functions to enable automatic detection and warning about the ECG signal quality. This can be accompanied by text and image information to help patients and caretakers (or a family member) to correctly operate the equipment.

- Deploying artificial intelligence (AI) techniques to automatically detect and warn about heart threatening arrhythmias and cardiac condition (e.g. detection of ST-segment alterations).

- Further testing in laboratory and on the field.

\section{Acknowledgment}

The authors are grateful to the volunteers who kindly authorized the recording of their ECGs using the developed ECG monitoring system using Android mobile and Bluetooth.

\section{References}

[1] http://www.who.int/mediacentre/factsheets/fs310.pdf (accessed 09.11. 2014).

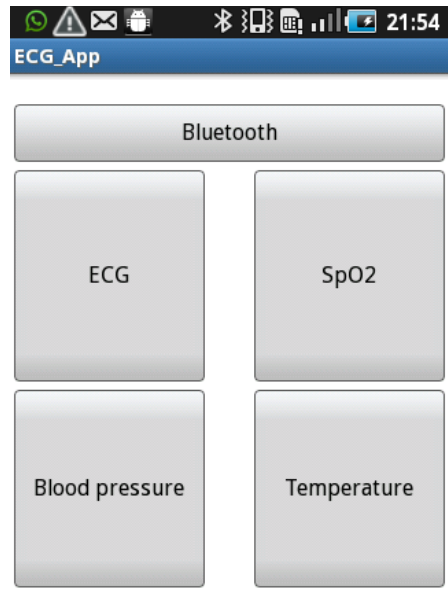

Fig. 5. First screen for the developed application.

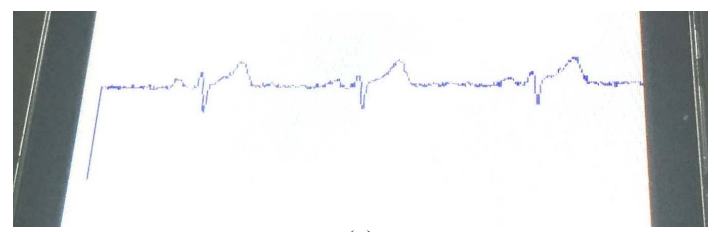

(a)

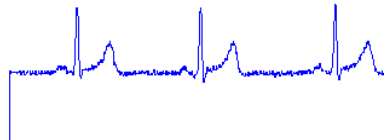

(b)

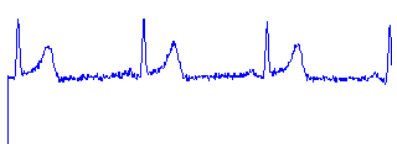

(d)

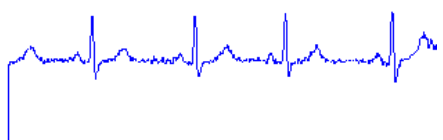

(c)

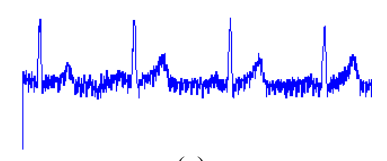

(e)
Fig. 6. ECG of volunteers (a) 1, (b) 2, (c) 3, and (d) 4; (e) ECG of one volunteer contaminated with noise.

[2] G. Lanza, "The electrocardiogram as a prognostic tool for predicting major cardiac events," Progress in Cardiovascular Diseases, v. 50, n. 2, 2007, pp. 87-111.

[3] N. Belgacem and F. Bereksi-Reguig, "Bluetooth portable device for ECG and patient motion monitoring," Nature \& Technology Review, n. 4, 2011, pp. 19-23.

[4] K. Liang, X. Zhang, Y. Wang, S. Huang, N. Guan, W. Pang, B. Li, and H. Chen, "A system of portable ECG monitoring based on Bluetooth mobile phone," International Symposium on IT in Medicine and Education (ITME), v. 2, 2011, pp. 309-312.

[5] Z. Yongning and Z. Guo, "A palm pilot based pocket ECG recorder," IEEE EMBS International Conference on Information Technology Applications in Biomedicine, 2000, pp. 110-112.

[6] M. Engin, Y. Yamaner, and Z. Engin, "A biotelemetric system for human ECG measurements," Measurement, v. 38, 2005, pp. 148-153.

[7] D. J. Harmah and D. Kathirvelu, "An ubiquitous miniaturized android based ECG monitoring system," (ICE-CCN) International Conference on Emerging Trends in Computing, Communication and Nanotechnology, 2013, pp. 117-120. 